\title{
Concept Analysis of Ethical Competence of Nursing Students and Nurses
}

\author{
Emi Yoshioka*, Sayuri Kaneko \\ Nagano College of Nursing, Komagane City, Japan \\ Email: *eyoshioka@nagano-nurs.ac.jp, kanekosa@nagano-nurse.ac.jp
}

How to cite this paper: Yoshioka, E. and Kaneko, S. (2019) Concept Analysis of Ethical Competence of Nursing Students and Nurses. Open Journal of Nursing, 9, 1173-1187. https://doi.org/10.4236/ojn.2019.911086

Received: October 28, 2019

Accepted: November 25, 2019

Published: November 28, 2019

Copyright $\odot 2019$ by author(s) and Scientific Research Publishing Inc. This work is licensed under the Creative Commons Attribution International License (CC BY 4.0).

http://creativecommons.org/licenses/by/4.0/

\section{cc) (i) Open Access}

\begin{abstract}
[Aim] This study aimed to conduct a concept analysis and clarification of ethical competence of nursing students and nurses, and further clarify the components. [Method] Rodger's concept analysis method was used. A literature search was conducted using the ICHUSHI database, MEDLINE, and CINAHL databases. The keywords included in the search were ethical competence. A total of 24 published articles were analyzed. [Results] As a concept of ethical competence among nursing students and nurses, five categories of [ethical sensitivity], [ethical reasoning], [ethical decision making], [ethical practice], [ethical reflection], obviously became. As a component, two categories of antecedent factors and three categories of consequences were identified. I defined ethical competence in nursing students and nurses, "including nursing students and nurses thinking processes and nursing practice and reflection to solve ethical problems Behavioral characteristics". [Conclusion] Review of nursing practice from an ethical perspective [ethical reflection] is indispensable for clarifying self-tasks and deepening better nursing practice.
\end{abstract}

\section{Keywords}

Ethical Competence, Nursing Student, Nurse, Concept Analysis

\section{Introduction}

With the rapid advancement and increasing complexity of medical care and development, as well as the establishment of comprehensive community care systems, nurses are now facing various new ethical issues. To address these issues, ethical judgment is required. In the "Code of Ethics for Nurses", the Japan Nursing Association clearly specifies guidelines for nurses' behavior and the scope of nurses' professional responsibilities [1]. In particular, for nurses to possess the ability to respond to ethical issues, the nurses' code of ethics suggests 
that all necessary knowledge should be provided during basic nursing education [1]. Many nursing students participate and practice in clinical settings; thus, such individuals require sufficient ethical competence even before their qualification as nurses. In response to this situation, the Ministry of Education, Culture, Sports, Science and Technology's (2011) bachelor's course in basic nursing education emphasizes ethics-related skills such as "the ability to defend the dignity and rights of patients" and "the ability to explain and obtain consent for nursing" as target abilities for nursing practice [2]. If nursing students begin working as nurses before learning ethical competence, they may encounter situations that they are unprepared to address; this can lead to a sense of helplessness, burnout, and career turnover. Thus, it is necessary for such students to systematically accumulate ethical competence, beginning in basic nursing education, and proceeding through continuing education.

Ethical competence has been defined as "the ability to appropriately resolve issues through applying ethics-related thinking and decision-making" [3], and also as "nurses implementing, based on subjective recognition, actions they feel to be most appropriate for the situation" [4]. Thus, ethical competence has been defined in different ways by researchers, and there remains no clear definition of its concepts and components. Therefore, in order to improve the ethical competence of nursing students and nurses by providing systematic nursing-ethics education, and to develop associated scientific and empirical research programs, it is first necessary to clarify the concept. Considering this, the present study aims to clarify the definition of ethical competence for nursing students and nurses, as well as its associated concepts and components.

\section{Methods}

\subsection{Research Method}

The approach used in the present study involves Rodgers' evolutionary concept analysis [5]. Rodgers' analysis is based on a philosophical perspective, suggesting that concepts are fluid and change with context, such as the passage of time and social background [5]. In the present study, we consider "ethical competence" to represent a concept that changes with alterations in medical and social environments; thus, we considered Rodger's approach to conceptual analysis to be suitable for this study.

\subsection{Data-Collection Method}

In educational psychology, behavior is considered an "competence," and in behavioral science knowledge and judgment are regarded as behavioral characteristics. For this reason, in the present study, we considered behavior to be based on knowledge and judgment, and chose as our keyword "ethical competence."

A literature search was conducted of EBSCO (specifically, the MEDLINE and CINAHL databases) and the ICHUSHI database. Domestic (i.e., Japan-based) studies were extracted from the ICHUSHI database, using "ethical competence" 
as the keyword, and without placing any limitation on the year of publication. Among the extracted documents, we read the abstracts and excluded reviews, articles concerning scale development, and documents that did not concern nursing students and nurses. Meanwhile, a search for overseas literature was performed using EBSCO (MEDLINE, CINAHL), again using "ethical competence" as the keyword and without limiting the year of publication. Among the extracted documents, we read the abstracts and excluded papers that were reviews, scale-development articles, and those that were not related to nursing students and nurses.

\subsection{Data-Analysis Method}

Based on Rodgers' evolutionary concept analysis method [5], we began by creating a data sheet to extract attributes that form the concept, the requirements necessary for the concept to exist, and descriptions of the consequences of the concept. Then, the extracted contents were coded and categorized.

Next, we examined the relationships between the categories relating to nurses and nursing students. Below, categories are presented by enclosing them in square brackets ([ ]).

\section{Results}

After performing, through a literature review, a conceptual analysis of ethical competence among nurses and nursing students using Rodgers' evolutionary concept analysis [5], we found that there were two categories of requirements, five categories of attributes, and three categories of consequences.

Below, the categories extracted as attributes, requirements, and consequences are presented by enclosing them in square brackets ([ ]), subcategories are enclosed in angle brackets $(<>)$, and the described literature is classified into whether it relates to nursing students or nurses (Table 1).

\subsection{Result of the Literature Search}

Through our keyword search, 28 domestic studies were initially returned; then, after applying the exclusion criteria, a total of six studies were extracted: three concerning nurses and three concerning nursing students. On the other hand, 87 nondomestic papers were returned, with 18 remaining after applying the exclusion criteria. These comprised 12 papers concerning nurses and six concerning nursing students.

Thus, in total we analyzed 24 cases.

\subsection{Antecedents}

As prior requirements, the categories [knowledge regarding ethical issues] and [opportunities to encounter ethical issues] were identified. These contained the subcategories $<$ knowledge required for ethical competence $>$ and $<$ skills required for ethical competence $>$, and $<$ problems relating to the situation of health $>$ and 
$<$ problems relating to situations regarding other health-care workers $>$, respectively.

Table 1. Antecedents, attributes, and consequences regarding nursing students and nurses' ethical competence.

\begin{tabular}{|c|c|c|c|c|}
\hline \multirow[t]{2}{*}{ Framework } & \multirow[t]{2}{*}{ Category } & \multirow[t]{2}{*}{ Subcategory } & \multicolumn{2}{|l|}{ Literature } \\
\hline & & & Nursing Students & Nurses \\
\hline \multirow{4}{*}{ Antecedents } & \multirow{2}{*}{$\begin{array}{l}\text { [Knowledge } \\
\text { regarding ethical } \\
\text { issues] }\end{array}$} & $\begin{array}{l}<\text { Knowledge required for ethical } \\
\text { competence }>\end{array}$ & \multirow[t]{2}{*}{ Vynckier et al., 2015} & $\begin{array}{l}\text { Chao et al., 2017; Tadd et al., 2006; } \\
\text { Karlsson et al., } 2010\end{array}$ \\
\hline & & $\begin{array}{l}<\text { Skills required for ethical } \\
\text { competence }>\end{array}$ & & Gjerberg et al., 2010 \\
\hline & \multirow{2}{*}{$\begin{array}{l}\text { [Opportunities to } \\
\text { encounter ethical } \\
\text { issues] }\end{array}$} & $\begin{array}{l}<\text { Problems relating to the situation of } \\
\text { health }>\end{array}$ & $\begin{array}{l}\text { Sakai et al., 2016; } \\
\text { Sinclair et al., } 2016\end{array}$ & Karlsson et al., 2017; Gjerberg et al., 2010 \\
\hline & & $\begin{array}{l}<\text { Problems relating to situation } \\
\text { regarding other health-care workers }>\end{array}$ & Sinclair et al., 2016 & Chao et al., 2017; Gjerberg et al., 2010 \\
\hline \multirow{12}{*}{ Attributes } & \multirow{2}{*}{ [Ethical sensitivity] } & $<$ Feeling discomfort $>$ & \multirow[t]{2}{*}{ Inoue et al., 2016} & \\
\hline & & $<$ Recognizing discomfort $>$ & & Yabushita et al., 2014 \\
\hline & \multirow{3}{*}{ [Ethical reasoning] } & $\begin{array}{l}<\text { Collecting information regarding } \\
\text { potential ethical situations }>\end{array}$ & & Karlsson et al., 2017 \\
\hline & & $\begin{array}{l}<\text { Examining situations recognized as } \\
\text { comprising an ethical issue }>\end{array}$ & $\begin{array}{l}\text { Inoue et al., 2016; } \\
\text { Sakai et al., } 2016\end{array}$ & Chao et al., 2017; Luz et al., 2016 \\
\hline & & $<$ Clarification of ethical issues $>$ & & Yabushita et al., 2014 \\
\hline & \multirow{2}{*}{$\begin{array}{l}\text { [Ethical } \\
\text { decision-making] }\end{array}$} & $<$ Determining the direction of care $>$ & Inoue et al., 2016 & Meyer et al., 2017; Yabushita et al., 2014 \\
\hline & & $<$ Clarification of behaviors $>$ & \multirow[t]{2}{*}{ Sakai et al., 2016} & \multirow[b]{2}{*}{ Jun, 2015; Yabushita et al., 2014} \\
\hline & \multirow{2}{*}{ [Ethical practice] } & $\begin{array}{l}<\text { Providing care in accordance with } \\
\text { judgements made regarding the } \\
\text { characteristics of the situation }>\end{array}$ & & \\
\hline & & $<$ Principles of nursing $>$ & $\begin{array}{l}\text { Inoue et al., 2016; } \\
\text { Sakai et al., 2016; } \\
\text { Uchida et al., } 2010\end{array}$ & $\begin{array}{l}\text { Chao et al., 2017; Karlsson et al., 2017; } \\
\text { Ooide, 2015; Wros et al., } 2004\end{array}$ \\
\hline & \multirow{3}{*}{ [Ethical reflection] } & $<$ Reviewing practice $>$ & \multirow[t]{2}{*}{ Sakai et al., 2016} & Jun, 2015; Poikkeus et al., 2013 \\
\hline & & $<$ Reviewing personal emotions $>$ & & Kim et al., 2016; Storaker et al., 2016 \\
\hline & & $<$ Learning from experience $>$ & Sakai et al., 2016 & \\
\hline \multirow{8}{*}{ Consequences } & \multirow{2}{*}{$\begin{array}{l}\text { [Improvement of the } \\
\text { quality of nursing } \\
\text { practice] }\end{array}$} & $\begin{array}{l}<\text { Improved ethical competence among } \\
\text { individuals }>\end{array}$ & Inoue et al., 2016 & $\begin{array}{l}\text { Chao et al., 2017; Luz et al., 2016; } \\
\text { Tobiyo et al., } 2012 \\
\text { Karlsson et al., } 2010\end{array}$ \\
\hline & & $\begin{array}{l}<\text { Improved ethical behavior in } \\
\text { departments }>\end{array}$ & Inoue et al., 2016 & Salmela et al., 2017 \\
\hline & \multirow{2}{*}{$\begin{array}{l}\text { [Development of } \\
\text { ethics] }\end{array}$} & $\begin{array}{l}<\text { Change in personal ethical } \\
\text { awareness }>\end{array}$ & \multirow[t]{2}{*}{ Uchida et al., 2010} & \\
\hline & & $<$ Change in personal ethical behavior $>$ & & Karlsson et al., 2017 \\
\hline & \multirow{4}{*}{$\begin{array}{l}\text { [Improvement } \\
\text { of autonomy as a } \\
\text { nursing professional] }\end{array}$} & & Dierckx et al., 1997; & Karlsson et al., 2010; Dierckx et al., 2008; \\
\hline & & $<$ Acting as a nursing professional $>$ & Trobec et al., 2014 & Karlsson et al., 2017 \\
\hline & & & & Poikkeus et al., 2014; Salmela et al., 2017 \\
\hline & & $\begin{array}{l}<\text { Taking responsibility for care, as a } \\
\text { nursing professional }>\end{array}$ & Sakai et al., 2016 & $\begin{array}{l}\text { Jun, 2015; Heikkinen et al., 2006; } \\
\text { Tadd et al., } 2006\end{array}$ \\
\hline
\end{tabular}


More specifically, [knowledge regarding ethical issues] was defined as learning the skills that serve as a foundation for ethical nursing practice. Sources of ethical-nursing-related information are lectures and exercises, which provide knowledge concerning ethical principles, the nursing code of ethics [6] [7], and ethical concepts [8]. Such sources also provide a means of continuing education and debate [9], and case studies allow students to familiarize themselves with the practices applied in decision-making [10].

Meanwhile, [opportunities to encounter ethical issues] was defined as exposure to situations in which nurses and nursing students encounter ethical problems during interaction with patients or with other health-care professionals. Ethical issues relating to patients include patient privacy and dignity [3], confidentiality [11], and communication [9]. Further, administering cardiopulmonary resuscitation to patients has also been reported to create an issue in this regard [10]. Meanwhile, ethical issues related to health-care workers include witnessing colleagues perform unsafe (or premature) medical practices [11] and the insufficient administering of care as a result of a lack of nurses [6] [9].

\subsection{Attributes}

Categories concerning attributes included [ethical sensitivity], [ethical reasoning], [ethical decision-making], [ethical practice], and [ethical reflection].

From these categories, 12 subcategories were extracted: <feeling discomfort $>$ and $<$ recognizing discomfort $>$ (for [ethical sensitivity]); <collecting information regarding potential ethical situations $>$, <examining situations recognized as comprising an ethical issue $>$, and $<$ clarification of ethical issues $>$ (for [ethical reasoning]); <determining the direction of care $>$ and $<$ clarification of behaviors $>$ (for [ethical decision-making]), <providing care in accordance with judgements made based on the characteristics of the situation $>$ and $<$ principles of nursing $>$ (for [ethical practice]), and $<$ reviewing practice $>$, $<$ reviewing personal emotions $>$, and $<$ learning from experience $>$ (for [ethical reflection]).

[Ethical sensitivity] was defined as developing an ability to notice, during daily nursing, aspects that are out of the ordinary and patient discomfort. This includes noticing incongruities (e.g., finding certain situations "strange" [12]), and recognizing discomfort, such as moistening [13].

[Ethical reasoning] was defined as collecting information related to unusual situations and clarifying the ethical issue by interpreting the needs and behaviors of each party involved (patients, family members, other health-care professionals, etc.). Examples from the extracted literature include: gathering information by applying a four-step approach [14], discussing why the nurse/nursing student felt a situation was "strange" [13], discussing the situation with others [3] [12], learning about the situation [3] [6] [15], and highlighting the relevant ethical issues.

[Ethical decision-making] was defined as making decisions regarding the direction of care and formulating specific action plans and clear priorities for re- 
solving ethical problems. In the papers we examined [12], feasible nursing [12] [13], utilizing ethical principles [13], and choosing approaches that are beneficial for patients [3] were mentioned. Additionally, one paper reported that decision-making was influenced by a lack of awareness of ethical issues [16].

[Ethical practice] was defined as providing planned and principles-based care in order to resolve ethical problems. The nurse provided the content discussed at the conference and the care deemed necessary for the patient [3] [13] [17]. In addition, we practice nursing according to the nursing code of ethics: respect and protect the human rights of patients [12] respond to patient needs [6] [18] [19]; communicate with patients and understand their issues [3] [12] [20]; provide explanations to patients and their families and, with their consent, help them [3]; be mindful of how information is managed [3] [14] [18]; and seek to provide safe and comfortable assistance for patients [3] [17].

[Ethical reflection] was defined as learning by considering personal feelings regarding ethical issues and reviewing ethical practices in terms of the ethical reasoning and ethical decision-making involved. Such consideration can include whether a judgment was correct [3], whether an action that was performed was based on ethical guidelines [21], and whether the action benefitted the patient [17]. In addition, sharing and discussing problems can alleviate mentally complex feelings [22] and help nurses/nursing students reconsider their related attitudes [18]. Furthermore, as a nurse, he learned seriously about the patient, reviewed the nursing provided, made an ethical decision, and learned to act with knowledge and skills [3].

Based on these five categories, the ethical competence of nursing students and nurses can be expressed as comprising the following: "The thinking process (ethical sensitivity, ethical reasoning, ethical decision-making) used to resolve ethical problems and to adopt appropriate behaviors, which include performing appropriate nursing practice and reflection."

\subsection{Consequences}

Categories concerning consequences comprised: [improvement of the quality of nursing practice], [development of ethics], and [improvement of autonomy as a nursing professional]. Here, six subcategories were extracted: <improved ethical competence among individuals $>$ and <improved ethical behavior in departments $>$; $<$ change in personal ethical awareness $>$ and $<$ change in personal ethical behavior $>$; and $<$ acting as a nursing professional $>$, <taking responsibility for care, as a nursing professional $>$, respectively.

[Improvement of the quality of nursing practice] was defined as fostering ethical competence in individuals and nursing departments. Through nursing, she gained the ability to grasp the main relationships associated with the problem [10]; further, by considering the patient's feelings, she developed a closer relationship with the patient [15] [23], and she fostered her ethical-reasoning ability through reflection [6]. There was also a workplace culture in which departments could easily consider ethical issues [12] [24]. 
[Development of ethics] was defined as a state in which ethics were transformed and refined through clinical experience. Through experience, I began to realize the appropriate attitudes for nurses and the importance of knowledge [20]. I also realized that I was able to think about ethics in the context of my nursing behavior [14].

[Improvement of autonomy as a nursing professional] was defined as practicing nursing in accordance with the code of ethics, and taking responsibility for personal actions. I thought I had to act as a member of nursing staff, adhering to established guidelines [21] [25] [26] [27], maintaining ethical-competence standards [24], and acting as a supporter of decisions [10] [14]. In addition, to assume responsibility for people's health, he had a responsibility to provide appropriate practice [3] [17] and thought he had to maintain high standards of ethical ability [7] [28].

\subsection{A Conceptual Model of Ethical Competence for Nursing Students and Nurses}

Based on the concepts identified through analysis of the requirements, attributes, and consequences, we developed a conceptual model (Figure 1).

First, as a pre-requisite, nursing students must learn ethical nursing through lectures and exercises; this relates to the category of [knowledge regarding ethical issues]. Knowledge [8], learning knowledge necessary for decision making [10]. In addition, in order to resolve ethical problems, nurses require a forum for discussion [9] and should strengthen their skills by conducting case studies [10]. Additionally, nursing students and nurses have opportunities to encounter ethical problems during their nursing practice, such as issues regarding patient privacy and dignity [3], a lack of confidentiality [11], and communication with patients [9].

When encountering ethical problems, nursing students and nurses noticed patient discomfort (such as something that seemed "strange"), which relates to the category of [ethical sensitivity] [12], and also felt personal discomfort, such as uncertainty [13]. Consequently, through [ethical reasoning], nurses collected information by applying a four-step method [14], discussed why they felt "strange" [13], discussed the situation with others [12] [13], obtained information regarding the cause of the issue [3] [6] [15], and reported the ethical problems. In [ethical decision-making], in order to resolve ethical problems nursing that accords with the code of ethics and ethical principles should be utilized [12] [13]. Further, the health-care team should collaboratively determine the most appropriate treatment methods for patients [3]. In accordance with the category of [ethical practice], meetings should be held regarding the necessary care for patients, and the approaches that are decided upon in these meetings should be implemented [13] [17], along with nursing that accords with the nursing code of ethics. Nursing students and nurses should conduct [ethical reflection] throughout the implementation of these four processes (ethical sensitivity, reasoning, decision-making, and practice). In other words, nursing students and 
Antecedents

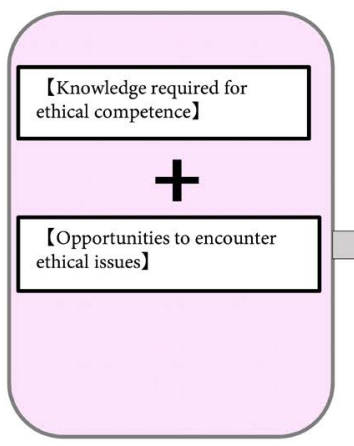

Attributes

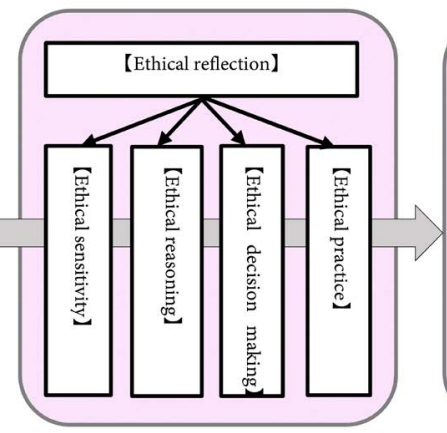

Consequence

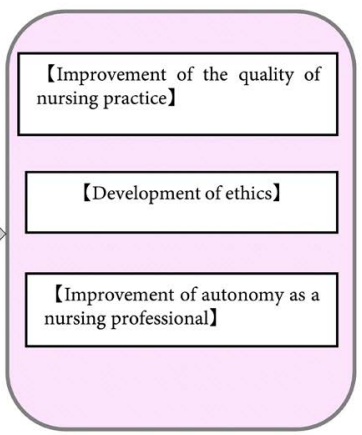

Figure 1. Model of ethical competence among nursing students and nurses.

nurses should perform reviews after each process in order to determine whether their judgments were correct [3], and whether they acted in accordance with ethical guidelines [21]. Moreover, they should also objectively consider whether their actions benefited the patient [17].

Through such an approach, [improvement of the quality of nursing practice] (for example, by fostering ethical sensitivity and ethical reasoning) is developed. Further, the ability to grasp the overall relationships related to such problems is enhanced and patients' feelings are improved; in particular, by considering patients' feelings, a closer nurse-patient relationship is fostered [2] [3] [15]. Meanwhile, ethical-reasoning ability is developed through reflection [6]. Changes also occur in regard to workplace culture, as departments more readily consider ethical issues [12] [24]. I felt that ethics was nurtured through experience, such as being able to consider ethics based on my nursing actions [14], this reflects the [development of ethics]. Furthermore, nursing professors should provide instruction that accords with existing guidelines [21] [25] [26] [27], emphasize ethical-competence standards [24], act as a supporter of decision support, etc. This relates to the category of [improvement of autonomy as a person].

\subsection{Commonalities and Differences between Nursing Students and Nurses Regarding Ethical Competence}

There were commonalities and differences between the nursing students and nurses regarding ethical competence.

Of the two subcategories of [ethical sensitivity], <discomfort> applied only to nursing students, while $<$ recognizing discomfort $>$ applied only to nurses.

Of the three subcategories of [ethical reasoning], <collecting information regarding potential ethical situations $>$ and $<$ clarifying ethical issues $>$ applied only to nurses, while <examining situations recognized as comprising an ethical issue $>$ applied to both nursing students and nurses.

Among the two subcategories of [ethical decision-making], $<$ determining the direction of care $>$ corresponded to both nursing students and nurses. On the other hand, <clarification of behaviors > applied only to nursing students. 
Of the two subcategories of [ethical practice], <providing care in accordance with judgements made regarding the characteristics of the situation $>$ applies only to nurses, while <principles of nursing $>$ applies to nursing students and nurses.

Of the three subcategories of [ethical reflection], <reviewing practice $>$ applied to both nursing students and nurses, <reviewing personal emotions $>$ applied only to nurses, and <learning from experience $>$ applied only to students.

\section{Discussion}

\subsection{Characteristics of the Concept of Ethical Competence among Nursing Students and Nurses}

Through the concept analysis conducted in the present study, we determined that the ethical competence of nursing students and nurses can be expressed as follows: "The thinking process (ethical sensitivity, ethical reasoning, ethical decision-making) used to resolve ethical problems and to adopt appropriate behaviors, which include performing appropriate nursing practice and reflection.”

Waithe [29] stated that ethical competence comprises 1) ethical sensitivity, 2) ethical reasoning, 3) attitudes, and 4) realization. However, in this conceptual analysis, we found the concept of "ethical reflection" to be an important feature of the concept of "ethical competence among nursing students and nurses." Schon [30] divides reflection into two categories: "reflection in action" and "reflection on action." Reflection in action" occurs in practice and affects decision-making and the provision of care. For the present study, this relates to nurses/nursing students considering whether a judgment is correct or whether ethical considerations have been implemented [3]. He looked back and forth and changed his subsequent behavior. In addition, he said that "reflection on actions" occurs after the event and contributes to the development of practical skills. In the present study, the actions to be taken were identified, and this led to concrete actions. Today, as medical care becomes more complex, various new ethical issues arise. For this reason, "ethical reflection," which involves reviewing nursing practice from an ethical perspective, is indispensable for clarifying issues relating to personal approaches and for improving nursing practice.

The analysis conducted in the present study showed that knowledge regarding ethical issues represents a foundation, and encountering ethical issues leads to ethical competence. Furthermore, in daily nursing, noticing incongruities (e.g., feeling something is "strange"), considering the cause, discovering behavior, practicing ethically and, through ethical reflection, fostering ethical competence, clarifies that ethical competence can be cultivated. The conceptual model based on these results indicates that basic nursing education provides opportunities to learn knowledge regarding nursing ethics and to acquire sufficient ethical competence to address the ethical problems that can be encountered in nursing practice. Notably, these results suggest that it is important for nursing-ethics education to emphasize ethical reflection. Furthermore, for continuous nursing educa- 
tion, constructing and providing nursing-ethics education that particularly emphasizes introspection, both for individuals and organizations, regarding ethical issues is suggested. The importance of constructing and providing nursing ethics education tailored to the latter stage was also suggested.

\subsection{Application of Ethical Competence for Nursing Students and Nurses, and Points to Note}

To illustrate how nursing students and nurses can practically demonstrate the concept of ethical competence defined in this analysis, in this section we provide example cases, and also list points that should be considered when applying ethical competence.

\subsubsection{Examples of the Application of Ethical Competence, Using Model Cases}

\section{1) Nursing student case}

Nursing student A is a fourth-year student at a nursing university [1]. In the second year of university, students were given instruction regarding ethical competence, which involved an explanation of nurses' ethical principles and the conducting of case studies using analytical tools to resolve ethical problems. In the third year of geriatric nursing practice, nursing student A witnessed nurse $\mathrm{C}$ apply a wheelchair restraint to patient $B$.

Nursing student A felt that nurse C's restraining of the patient was unusual, and felt discomfort regarding the incident. After examining the patient's electronic medical record, nursing student $\mathrm{A}$ found that patient $\mathrm{B}$ had dementia and a history of falls, and also that the patient had osteoporosis, meaning there was a high risk of fracture from falls. Nursing student A now understood nurse C's decision to restrain the patient. However, nursing student A still felt that the physical restraint limited patient B's activities and motion, and could also cause physical and mental pain; consequently, nursing student A sought to find a less restrictive method of protecting patient B. In particular, nursing student A thought that patient B must have a reason for trying to leave the wheelchair, so he asked the patient where he wanted to go, and helped him reach his goal. As a result of this approach, patient $B$ ceased trying to leave the wheelchair. Nursing student A objectively reviewed his judgments and actions at each stage of the process, examining whether his judgments were correct, whether ethical consideration was implemented, and whether the best assistance was being implemented. In addition, by discussing at a meeting whether patient $B$ was safe after the care was administered, it was possible to identify the approaches that most benefitted the patient.

Nursing student A felt that patient B's safety and comfort were ensured by applying an approach that involved respecting patient B's dignity while considering how to protect his safety, and by then implementing nursing that was tailored to his personal characteristics. Nursing student A felt that nurse C's controlling of the patient was unusual, so he analyzed his personal feelings regard- 
ing the situation, clarified the relevant care policy, and confirmed whether patient B's safety could be protected in a less restrictive manner. As a result of the discussions at the health-care team's meeting, the nursing student felt that not only his own ethical competence, but that of all students present, was strengthened. Nursing student A felt that it was important for nurses to consider solutions and act ethically instead of ignoring situations that caused them discomfort. In the future, when I feel a sense of incongruity, I plan to act independently.

\section{2) Nurse's case}

Nurse D is a nurse with three years of experience. At university, he was taught the knowledge necessary for ethical competence, such as the code of ethics and ethical principles for nurses, and attended ethics conferences during nursing practice. Furthermore, during the training he received as a new employee at the hospital, he learned of ethical problems that are likely to occur in hospitals, and examined real cases of ethical problems, applying a four-step approach to resolve them.

One day, during practice, Nurse D noticed that a colleague, Nurse E, left an electronic medical record in the hospital room.

Nurse D felt that electronic medical records should not be left in hospital rooms that nonmedical personnel can freely access. Investigating why the electronic medical record was left in the patient's room, Nurse D found that Nurse E had left the room to respond to another patient's call for a nurse. Nurse D felt that nurses should be responsible for protecting patients' personal information at all times, regardless of the situation; however, he also felt that this was a possible case when a sudden response was required on the part of Nurse E. Nurse D thought that this was an issue that should be addressed by the organization, not by himself, in order to protect the patient's personal information, and he had an opportunity to consult and discuss with the nurse. At the ward meeting, nurse D explained the situation and the need to protect patients' personal information. If he left the electronic medical record in the ward for a short time, he was overconfident that there was no information leakage. Some of the nurses present stated that they did not think this was important, that they had a hard time, or that they already made conscious efforts to protect personal information. As a result of the conference, opinions such as returning the PC to the nurse station, logging out of the PC, and requesting other nurses take responsibility for the record were suggested, and an organizational policy was consequently decided. After the conference, the electronic medical records were no longer left in rooms, and nurses spoke to each other to protect patients' personal information. Nurse D objectively reviewed his judgment and behavior at each stage of the process and considered whether the organization's judgment was correct and whether there was an appropriate means of protecting patients' personal information. In addition, he was able to reexamine his ethical competence as a nurse by discussing the issue at the meeting and collaborating in the decision-making regarding whether patients' personal information could be protected. 
Nurse D thought it was unusual that a patient's personal information was not protected. By raising and discussing the problem at the ward meeting, the policy was consequently clarified and individual nurses were given instruction regarding how to act autonomously to protect personal information. He felt that not only his own ethical competence, but that of the entire ward was strengthened. When Nurse D felt discomfort regarding the practice he had witnessed, he felt that it was important for nurses to consider solutions and to act ethically. In the future, when I felt a sense of incongruity in my daily practice, I wanted to work actively with the organization and act ethically.

\subsubsection{Points to Note When Applying the Concept Model}

The characteristics of nursing ethics cause the commonalities and differences that exist between nursing students and nurses regarding ethical competence.

Ethical ability develops continuously over time rather than remaining at a mere minimum standard, and even students need to possess a comprehensive degree of ethical ability. In other words, both students and nurses continue to develop their ethical competence, and students should receive training in this behavioral characteristic. Varying levels of competence may be the reason nursing students and nurses are perceived as having differing ethical competencies. There are three types of behaviors in this regard: action-based (skill-dependent), rule-based, and knowledge-based. These three performance levels are influenced by both psychological (conscious, hybrid, automatic) and situational factors (novel problems, problems for which nurses have received training, routines). An overview of ethical competence in regard to these three behavior types shows that nursing students transition from a skill base based on conscious action to a mixed-rule base, while nurses are moving from a mixed rule base, which resembles an automated knowledge base. Indeed, in [ethical practice], students are consciously thinking of and acting to address novel problems based solely on knowledge and rules they have learned, because they have little experience; this is considered to be a mixed-rule base from a knowledge base that acts in accordance with. On the other hand, because nurses have experience of various situations, they act not only according to rules, but also provide $<$ care in accordance with judgements made regarding the characteristics of the situation>, which can be considered to represent an automated knowledge base.

Based on the above, we believe that this concept model should only be applied after considering the commonalities and differences between nursing students and nurses.

\subsection{Limitations to This Study}

Of the 24 articles examined in this study, only six were domestic. Ethical competence is affected by cultural environment and background; therefore, it is necessary to more accurately verify whether this concept model applies to the ethical competence of nurses and nursing students in Japan. In order to verify this, future studies should evaluate, based on the analysis result of this concept, 
whether the ethical competence of nurses and nursing students is promoted through nursing ethics education and the application of specific measures for nursing ethics education.

\section{Conclusion}

Ethical competence among nursing students and nurses is defined as "the thinking process (ethical sensitivity, ethical reasoning, ethical decision-making) used to resolve ethical problems and to adopt appropriate behaviors, which include performing appropriate nursing practice and reflection". The ethical behavior of nurses and nursing students comprises five attributes: [ethical sensitivity], [ethical reasoning], [ethical decision-making], [ethical practice], and [ethical reflection]. It also comprises two preceding requirements: [knowledge regarding ethical issues] and [opportunities to encounter ethical issues]. As a consequence, three were shown, in other words, [Improvement of quality of nursing practice] [Development of ethics] and [Improvement of autonomy as a nursing professional].

\section{Funding}

This research was conducted with the assistance of a Grants-in-Aid for Scientific Research, Grant C (general number 17K12134).

\section{Conflicts of Interest}

The authors declare no conflicts of interest regarding the publication of this paper.

\section{References}

[1] Japan Nursing Association (2003) Code of Ethics for Nurses. https://www.nurse.or,jp/home/publication/pdf/rinri/code_of_ethics.pdf

[2] Ministry of Education, Culture, Sports, Science and Technology (2012) Nursing Practice Skills and Goals to Be Achieved at the Time of Graduation, Which Represent the Core of the Undergraduate Program. http://www.mext.go.jp/b_menu/shingi/chousa/koutou/47/siryo/_icsFiles/afieldfile/ 2011/11/04/1312488_5.pdf

[3] Sakai, M., Kawai, T. and Koide, E. (2016) The Attitudes of Nurses Who Can Perform Ethical Behavior, as Captured by Students Learning from Case Analyses of Ethical Problems. Co-Creation Welfare, 11, 37-46.

[4] Chichi, A. and Kimiko, N. (2017) Characteristics of Organizational Culture in which Nurses Show High Levels of Ethical Behavior. Bulletin of Mie Prefectural College of Nursing, 21, 1-9.

[5] Rodgers, L. and Knafl, A. (2000) Concept Development in Nursing: Foundations, Techniques, and Applications. 2nd Edition, Saunders, Philadelphia, PA.

[6] Chao, S., Chang, Y., Yang, C. and Clark, J. (2017) Development, Implementation, and Effects of an Integrated Web-Based Teaching Model in a Nursing Ethics Course. Nurse Education Today, 55, 31-37.

https://doi.org/10.1016/j.nedt.2017.04.011 
[7] Tadd, W., Clarke, A., Lloyd, L., Leino-Kilpi, H., Strandell, C., Lemonidou, C., Heymans, R., et al. (2006) The Value of Nurses' Codes: European Nurses' Views. Nursing Ethics, 13, 376-393. https://doi.org/10.1191/0969733006ne891oa

[8] Vynckier, T., Gastmans, C., Cannaerts, N. and Casterle, B. (2015) Effectiveness of Ethics Education as Perceived by Nursing Students: Development and Testing of a Novel Assessment Instrument. Nursing Ethics, 22, 287-306. https://doi.org/10.1177/0969733014538888

[9] Gjerberg, E., Forde, R., Pedersen, R. and Bollig, G. (2010) Ethical Challenges in the Provision of End-of-Life Care in Norwegian Nursing Homes. Social Science \& Medicine, 71, 677-684. https://doi.org/10.1016/j.socscimed.2010.03.059

[10] Karlsson, M., Roxberg, A., Silva, A. and Berggren, I. (2010) Community Nurses' Experiences of Ethical Dilemmas in Palliative Care: A Swedish Study. International Journal of Palliative Nursing, 16, 224-231. https://doi.org/10.12968/ijpn.2010.16.5.48143

[11] Sinclair, J., Papps, E. and Marshall, B. (2016) Nursing Students' Experiences of Ethical Issues in Clinical Practice: A New Zealand Study. Nurse Education Practice, 17, 1-7. https://doi.org/10.1016/j.nepr.2016.01.005

[12] Inoue, N., Yoshidome, A., Wakamatsu, M., et al. (2016) Recognition of Ethics Conferences in Nursing Practice. Analysis of Ethics Conference Records in Maternity Nursing Practice, 8, 3-15.

[13] Yabushita, Y., Takekawa, Y. and Mochimochi, C. (2014) Evaluation of Trials of Ethical Nursing Practice Support for Nurses Specializing in Chronic Disease Nursing. Bulletin of Faculty of Nursing, Osaka Prefecture University, 20, 77-83.

[14] Karlsson, M., Karlsson, N. and Hilli, Y. (2017) Ethical Dilemmas during Cardiac Arrest Incidents in the Patient's Home. Nurse Ethics, 26, 625-637. https://doi.org/10.1177/0969733017709337

[15] Luz, K., Vargas, M.A., Barlem, L., Schmitt, P., Ramos, F. and Meirelles, B. (2016) Coping Strategies for Oncology Nurses in High Complexity. Revista Brasileira Enfermagem, 69, 59-63. https://doi.org/10.1590/0034-7167.2016690109i

[16] Meyer-Zehnder, B., Albisser Schleger, H., Tanner, S., Schnurrer, V., Vogt, D.R., Reiter-Theil, S. and Pargger, H. (2017) How to Introduce Medical Ethics at the Bedside-Factors Influencing the Implementation of an Ethical Decision-Making Model. BMC Medical Ethics, 18, 16. https://doi.org/10.1186/s12910-017-0174-0

[17] Jun, O. (2015) Relationship between Nurses' Behavioral Choices in Conflict Situations and Perceptions of Ethical Behavior. Journal of Fujieda City General Hospital, 21, 15-20.

[18] Storaker, A., Naden, D. and Saeteren, B. (2016) From Painful Busyness to Emotional Immunization: Nurses' Experiences of Ethical Challenges. Nursing Ethics, 24, 556-568. https://doi.org/10.1177/0969733015620938

[19] Wros, P., Doutrich, D. and Izumi, S. (2004) Ethical Concerns: Comparison of Values from Two Cultures. Nursing \& Health Sciences, 6, 131-140. https://doi.org/10.1111/j.1442-2018.2004.00184.x

[20] Uchida, F., Nishioka, M., Tamura, M., et al. (2010) Self-Image About the Human Nature as a Health Care Professional that Students Perceive in Basic Nursing Practice II. Journal of Health Science, 10, 176-182.

[21] Poikkeus, T., Leino-Kilpi, H. and Katajisto, J. (2014) Supporting Ethical Competence of Nurses during Recruitment and Performance Reviews-The Role of the Nurse Leader. Journal of Nursing Management, 22, 792-802.

https://doi.org/10.1111/jonm.12033 
[22] Kim, S., Seo, M. and Kim, D. (2016) Unmet Needs for Clinical Ethics Support Services in Nurse: Based on Focus Group Interviews. Nursing Ethics, 25, 505-519. https://doi.org/10.1177/0969733016654312

[23] Tobiyo, T. and Sakai, K. (2012) Nurses' Awareness of Ethics Conferences. Journal of Japan Society for Nursing Ethics, 4, 15-22.

[24] Salmela, S., Koskinen, C. and Eriksson, K. (2017) Nurse Leaders as Managers of Ethically Sustainable Caring Cultures. Journal of Advanced Nursing, 73, 871-882. https://doi.org/10.1111/jan.13184

[25] Dierckx, B., Izumi, S., Godfrey, N. and Denhaerynck, K. (2008) Nurses' Responses to Ethical Dilemmas in Nursing Practice: Meta-Analysis. Journal of Advanced Nursing, 63, 540-549. https://doi.org/10.1111/j.1365-2648.2008.04702.x

[26] Dierckx, B., Grypdonck, M., Vuylsteke-Wauters M. and Janssen, P.J. (1997) Nursing Students' Responses to Ethical Dilemmas in Nursing Practice. Nursing Ethics, 4, 12-28. https://doi.org/10.1177/096973309700400103

[27] Trobec, I. and Starcic, A.I. (2015) Developing Nursing Ethical Competences Online Versus in the Traditional Classroom. Nursing Ethics, 22, 352-366. https://doi.org/10.1177/0969733014533241

[28] Heikkinen, A., Lemonidou, C. and Petsios, K. (2006) Ethical Codes in Nursing Practice: The Viewpoint of Finnish, Greek and Italian Nurses. Journal of Advanced Nursing, 55, 310-319. https://doi.org/10.1111/j.1365-2648.2006.03912.x

[29] Waithe, E. (1989) Developing Case Situations for Ethics Education in Nursing. Journal of Nursing Education, 28, 175-180.

[30] Schon, D. (1991) The Reflective Practitioner. 2nd Edition, Jossey Bass, San Francisco, CA. 